\title{
Gender and Cultural Differences in Perceiving Game Characters of Digital Educational Games
}

\author{
Effie Lai-Chong Law ${ }^{1}$, Tim Gamble ${ }^{1}$, and Daniel Schwarz ${ }^{2}$ \\ ${ }^{1}$ Departmen of Computer Science, University of Leicester, LE1 7RH, UK \\ ${ }^{2}$ TAKOMAT GmbH, Neptunplatz 6b, 50823 Köln, Germany \\ \{elaw, gamble\}@mcs.le.ac.uk, dan@takomat.com
}

\begin{abstract}
A survey on the initial design of a digital educational game was developed and administered to the target group in Germany and England. Some significant gender and cultural differences in game play habit, game type preferences and game character perceptions were observed.
\end{abstract}

\section{Introduction}

Developing digital educational games (DEGs) that can cost-effectively foster learning with fun and pleasure is a vision for researchers and practitioners in the field of HCI and technology-enhanced learning. DEGs offer exciting and dynamic environments which engage players in meaningful and motivating learning activities, inspiring them to explore a variety of topics and tasks. Nonetheless, previous research suggests that children in general tend to find educational games uninteresting, and that gender difference existed with boys holding a more negative attitude towards edutainment games than girls [1]. Interestingly, while the number of girls playing computer games has been increasing, they still tend to be perceived as masculine activity that more boys than girls prefer and spend time on. Such a disparity is attributable to the stereotypical presentation within games and to a general lack of female characters in games [2]. Even children in elementary schools perceive that software is gendered by design. The implication is more than just the attitude towards games; more serious impacts are girls' low confidence in working with computers and avoidance from technologyrelated fields, adversely affecting their employability. Specifically, Kinzie and Joseph [1] identified some interesting gender issues in game character preferences, for instance, the children in their study preferred characters to be of their same gender and ethnicity. Presumably, culture with its values, beliefs and norms plays an important role in shaping children's perceptions of game characters.

We are motivated to study gender and culture differences in the context of a DEG under development. The prototype topic is based on geography. An initial game design concept, prior to any implementation, was developed. Put briefly, the game story was about an alien kidnapping a boy and their flying round the world to collect relevant geographical information. A survey was designed with three objectives: (i) to evaluate the acceptance of the representative users of the game towards the game design; (ii) to evaluate if there are any gender and cultural differences in perceiving 
the game characters; (iii) to elicit feedback on improving the game concept; a practical means to gather user requirements. It was administered in two countries.

\section{Method and Procedure}

Design of the Questionnaire. The questionnaire consists of two major parts. Part A contains five close-end questions on the respondent's gender, age, gameplay habit, gametype preference, and affinity for geography. Specifically, four gametypes learning, action, strategic and sport - are provided as options to reduce the possible confusion in children; the other taxonomies are deemed rather complex (e.g. [3]). Part $\mathrm{B}$ addresses different aspects of the game. First a synopsis of the game story is presented. Then two close-end questions on the perceived interestingness of stories about aliens/UFO in general and of the game story in particular. An open-end question on describing improvement suggestions is presented. A set of four questions on understanding how respondents identify themselves with the story's main play characters are given. Another set of three questions on the preference of non-play character is posed. The last question is to assess the respondent's intention to play the game in the future.

Participants. Two samples from Germany and England were involved in the survey. They were school children aged between 11 and 14, the target group of the game. In Germany, the survey was conducted in the context of computer games fair. In England, the survey was administered in the classrooms of the five participating schools. Due to organizational constraints, the survey could only be conducted by the school teachers, who were asked to read aloud a script with similar wordings used in the German event. This step was taken to maximize the comparability of the data collected from the two settings.

Table 1. Demographic data of the survey respondents in the two countries

\begin{tabular}{|l|l|c|c|c|}
\hline Country & Number/Age & Girls & Boys & Sub-total \\
\hline German & Number & 78 & 61 & 139 \\
& Mean Age (SD) & $12.6(1.1)$ & $12.8(1.1)$ & $12.7(1.1)$ \\
\hline \multirow{2}{*}{ British } & Number & 59 & 83 & 142 \\
& Mean Age (SD) & $12.5(0.9)$ & $12.7(0.9)$ & $12.6(0.9)$ \\
\hline & Sub-total & 137 & 144 & 281 \\
\hline
\end{tabular}

\section{Results, Discussions and Concluding Remarks}

Results show that half of the British boys (52\%) play games everyday and half of the German boys $(51 \%)$ play games more than twice per week. Interestingly, $14 \%$ and $12 \%$ of the British and German girls report that they have never played games, whereas all of the British boys have played games. $45 \%$ of the German girls play games less than once per week whilst $44 \%$ of their British girls play more than twice per week. These figures seem to suggest that (i) Boys tend to play games more 
frequently than girls, irrespective of the country of residence; (ii) the British children tend to play games more frequently than their German counterparts. To investigate whether these observations are statistically significant, we performed the linear categorical regression analysis. The value of $R^{2}=.25$ indicates that the two predictor variables gender and country can explain only $25 \%$ of the variations of the gameplay frequencies. Results show the significant effect of the predictor gender (beta $=.49$, $t=9.32, p<.001)$ ) and the non-significant effect of the covariate country (beta $=-.017$, $t=-.136, p>0.05)$. Boys tend to play games more frequently than girls, and the country of residence does not have a strong effect on the children's gameplay frequency.

Cramer's V was used to evaluate if gender was associated with gametype preferences. The most preferable gametype for both the British girls (51.7\%) and boys $(49.5 \%)$ are Action, followed by Strategic and Sport. The least preferable gametype is Learning with only $3.2 \%$ and $2.2 \%$ for the girls and boys, respectively. The value of the Pearson chi-square equals $0.581(p=.901)$, indicating that gender and gametype preference for the British sample are not significantly related. In contrast, the German sample demonstrates a slightly different pattern from their British counterparts. The most preferable gametype for the German girls is Strategic (40.7\%), followed by Action and then Sport; the most preferable gametype for the German boys is Action (54.3\%), followed by Strategic and then Sport. The least preferable gametype is Learning with $13.2 \%$ and $3.2 \%$ for the girls and boys, respectively. The value of the Pearson chi-square equals $13.972(p=.003)$, indicating that gender and gametype preference for the German sample are significantly related.

With the aim of evaluating to what extent the respondents tended to associate the Boy's (the main play character) attributes with their own, they were asked to rate first the Boy and then themselves, using a 7-point scale, with respect to six pairs of contrasting adjectives adapted from the instrument Speech Evaluation Instrument [4] consisting of three subscales - superiority, attractiveness and dynamism, against which the entity of interest is evaluated:

- Superiority: Intelligent vs. Unintelligent; Uneducated vs. Educated;

- Attractiveness: Friendly vs. Unfriendly; Cold vs. Warm;

- Dynamism: Peaceable vs. Aggressive; Talkative vs. Shy

The exercises resulted in a set of so-called "Boy-based ratings" and another set of "Me-based ratings". We computed the correlations among them independently for the German and British samples. A number of statistically significant correlations are found. Nonetheless, based on our research interest, we explore to see whether there are gender differences in perceiving the relationships between the Boy's attributes, between the Me attributes, and between these two sets. Interestingly, results consistently show that the German female respondents tended to perceive the attribute interrelations, be they applied to the Boy or themselves, in a more complicated manner than did their male counterparts. Presumably, the German male respondents may associate their own attributes with the Boy's (same gender) more strongly than the female respondents (opposite gender) do; the empirical results indicate otherwise. In contrast, the British respondents' perceptions, irrespective of gender, are less 
complicated than those of their German counterparts. Interestingly, the British male respondents tend to perceive the associations in a more complex way than their female ones - a reversed trend demonstrated by the German sample. Fig. 1 illustrate the results how the respondents perceive the associations between the game main play character ("Boy") and themselves ("Me"). Contrasts are observed across gender and culture. We also aim to find out whether those who perceived a stronger "Boy-Me" association might have a higher tendency to play the game in the future (i.e. the last question of the survey) by summing the absolute differences in ratings over the six pairs of adjectives. While there is a moderately significant correlation for the British sample $(\mathrm{r}=-.24, \mathrm{~N}=199, p<.05)$, it is not significant for the German sample.

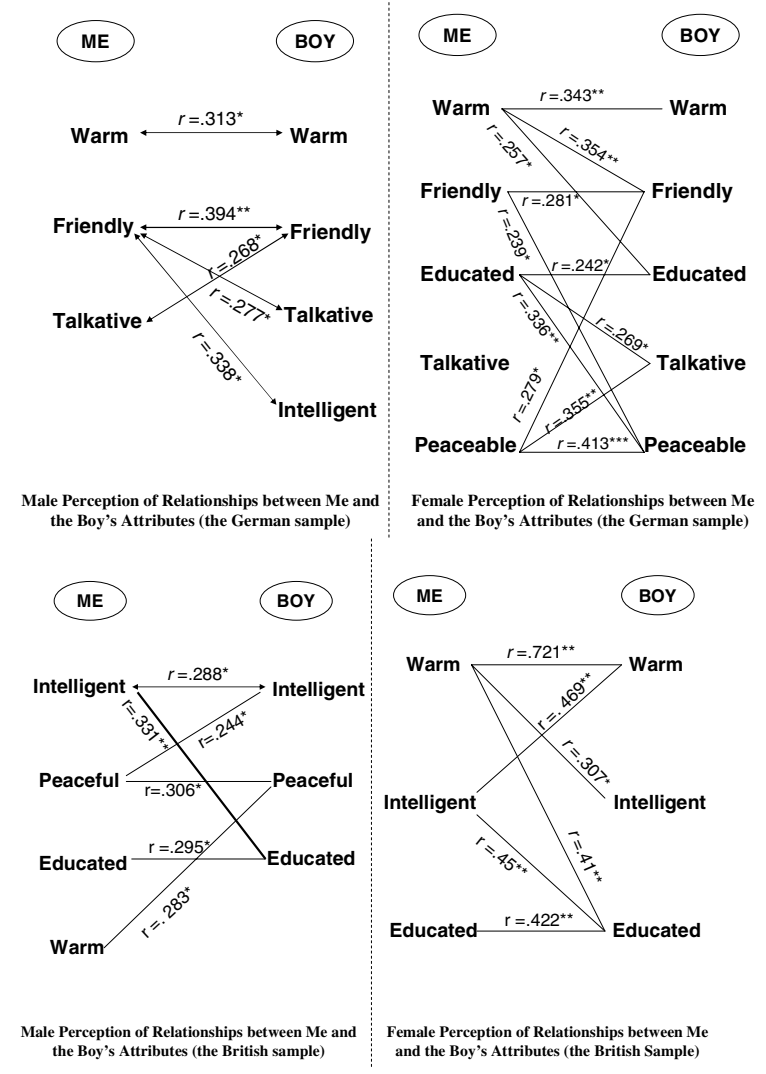

Fig. 1. The British (left) and German (right) children perceptions of the main game character. On both diagrams, boys are on the left and girls on the right.

Concluding Remarks: Previous research suggests that children, especially boys, tend to find learning games boring. It is corroborated by our findings that among the four gametypes learning game is least preferable and girls are more positive towards it than boys. Existing research also suggest that children tend to prefer game characters that are in some way "like me". Cultural preferences for normative personal qualities may influence children's preferences for the characters they play. While there are some very interesting gender and cultural differences in interpreting the main play character's qualities and in associating those qualities to theirs, such associations do not affect their intention to play the game. The setting where the survey was conducted could have impact on the children's perception and acceptance of the game: the relaxing atmosphere in the game fair with the exhibitors as opposed to the more structured classroom environment with the teacher. We explore psychosocial theories to explicate the phenomena observed and their implications on future work. 


\section{References}

1. Kinzie, M.B., Joseph, D.R.D.: Gender differences in game activity preferences of middle school children. Educational Technology Research \& Development 56, 643-663 (2008)

2. Gentry, M., Gable, R.K., Rizza, M.G.: Students' perceptions of classroom activities: Are there grade-level and gender differences? Journal of Educational Psychology 94(3), 539544 (2002)

3. Apperley, T.H.: Genre and game studies: Toward a critical approach to video game geners. Simluation \& Gaming 37(1), 6-23 (2006)

4. Zahn, C.J., Hopper, R.: Measuring language attitudes: The speech evaluation instrument. Journal of Language and Social Psychology 4, 113-122 (1985) 